\title{
A Field study to Estimate the Prevalence of Bovine African Trypanosomosis in Butaleja District, Uganda
}

\author{
Zhang JING ${ }^{1)}$, Joseph W. MAGONA ${ }^{2,3)}$, Tatsuya SAKURAI ${ }^{1)}$, Oriel M. M. THEKISOE ${ }^{1)}$, Charles P. OTIM ${ }^{3)}$, \\ Chihiro SUGIMOTO ${ }^{2)}$ and Noboru INOUE ${ }^{1) *}$ \\ ${ }^{1)}$ National Research Center for Protozoan Diseases, Obihiro University of Agriculture and Veterinary Medicine, Inada-cho, Obihiro, \\ Hokkaido 080-8555, ${ }^{2)}$ Research Center for Zoonosis Control, Hokkaido University, Sapporo, Hokkaido 060-0818, Japan and \\ 3) National Livestock Resources Research Institute (NaLIRRI) P.O. Box 96 Tororo, Uganda
}

(Received 24 July 2008/Accepted 3 December 2008)

ABSTRACT. Prevalence of bovine trypanosomosis was determined from a total of 203 blood samples collected from Butaleja district, eastern Uganda. All samples were examined by microhematocrit centrifuge test (MHC), PCR and ELISA. ELISA was performed in accordance with the OIE standard procedures using Trypanosoma brucei gambiense procyclic form crude antigens. PCR were utilized to identify the species and the subspecies of trypanosome. The overall prevalence of bovine African trypanosomosis was $8.9 \%$ by MHC, and $45.3 \%$ by the ELISA. Since substantial number (12 out of 18 ) of MHC positive samples were negative in the PCR tests, we could not conclude the most epidemic trypanosome species in the studied area. Nevertheless, the PCR results suggests that the most prevalent trypanosome was $T$. $b$. brucei (31/203), followed by $T$. congolense (6/203). In addition, only a few (3/203) mixed infections of $T$. $b$. brucei and T. congolense was detected by the PCR. Results obtained from this study indicates that bovine trypanosomosis is endemic in Butaleja district, Uganda.

KEY WORDS: bovine, prevalence, trypanosome, Uganda, zoonotic parasite.

African trypanosomosis in cattle is caused by protozoan parasites of the genus Trypanosoma, including T. brucei, $T$. congolense, and T. vivax. These trypanosomes are transmitted by tsetse flies of the genus Glossina [4, 8]. Bovine trypanosomosis is a chronic debilitating disease causing severe cachexia and anemia with associated intermittent fever, edema and loss of condition. The disease is frequently fatal and is a major constraint to livestock and agriculture production in Africa. Accordingly, trypanosomosis is ranked among the top of 10 global cattle diseases impacting on the poor community [3]. Infection with pathogenic trypanosome species affect various aspects of cattle productivity, often resulting in death.

The aim of the present study was to determine the prevalence and a major causative agent of bovine trypanosomosis in Butaleja district, eastern Uganda (Fig. 1). ELISA was performed to detect trypanosome specific antibodies from 203 bovine samples, whilst microhematocrit centrifuge test (MHC) and PCR were also carried out to determine level of parasitemia and trypanosome species and sub-species infecting the sampled cattle, respectively. All the cattle blood samples were collected in Butaleja district, eastern Uganda (Latitude $0^{\circ} 54^{\prime} \mathrm{N}$, Longitude 3357' E, Altitude $1,071 \mathrm{~m}$ ) in the early dry season (July, 2006) with temperatures ranging from 23 to $35^{\circ} \mathrm{C}$. Poor pasture conditions and high temperatures cause nutritional stress and lower hematocrit value, whereby diseases incidence in cattle tends to

\footnotetext{
* Correspondence to: Dr. Inoue, N., National Research Center for Protozoan Diseases, Obihiro University of Agriculture and Veterinary Medicine, Inada-cho, Obihiro, Hokkaido 080-8555, Japan.

e-mail: ircpmi@obihiro.ac.jp
}

increase in the dry season [19]. Glossina fuscipes fuscipes is the dominant tsetse species in the area with scanty G. pallidipes [11]. In addition, biting flies such as tabanids also exist in the area. Most people in the area are smallholder farmers keeping cattle, goats, pigs, and sheep integrated with crop production. Cattle, mainly of the Zebu breed, are kept under traditional communal grazing management system $[9,12]$. Blood samples were collected from jugular vein

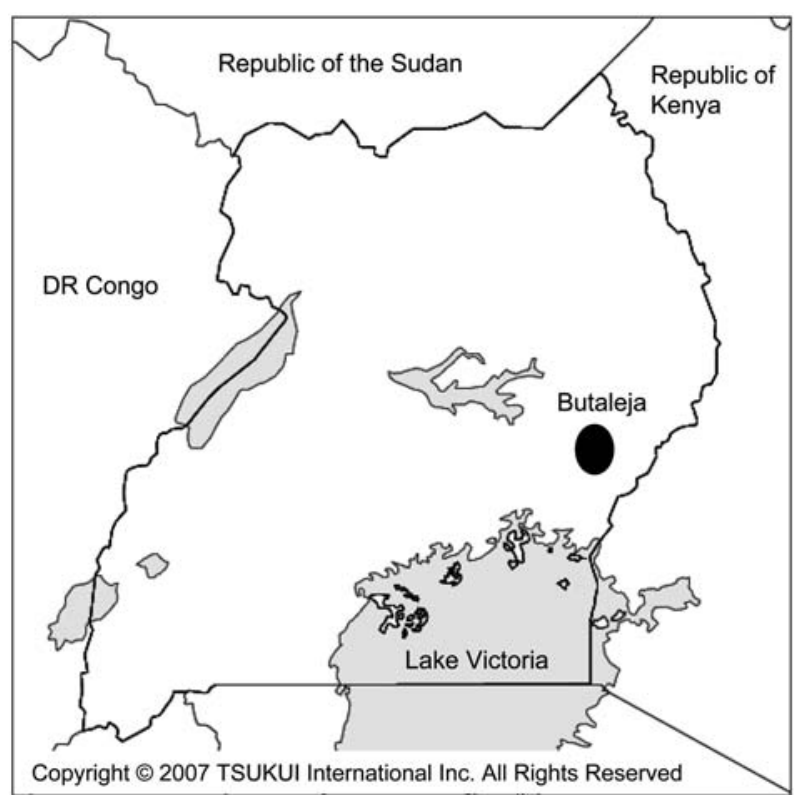

Fig. 1. Map of Uganda showing the location of Butaleja district (Closed circle). 
of each animal into EDTA treated and plain vacutainer tubes. Samples were examined by MHC at the site of sampling, and serum and total blood DNA was isolated at National Livestock Resources Research Institute (NaLIRRI). MHC was conducted in accordance with the OIE manual of standards for diagnostic tests and vaccines [17]. Total blood DNA was extracted using DNA extraction kit (Cat. No. 9081, TaKaRa Bio Inc. Japan) according to manufactures instructions. Trypanosome crude antigen for antibody detection ELISA was prepared as described in the OIE manual [17]. Briefly, procyclic form (PCF) of T. $b$. gambiense IL2343 was propagated in vitro. PCF cells were collected and washed 3 times with PBS. The trypanosome suspension was subjected to 7 freeze and thaw cycles and centrifugation at 10,000 rpm for $30 \mathrm{~min}$. The supernatant was collected and used as T. b. gambiense PCF crude antigen. The cut-off value of the ELISA was based on the mean optical density (OD) value (+ 3 standard deviation) obtained from testing 30 trypanosome negative sera from cattle of a non-endemic area (around Obihiro, Japan). As a result, the cut-off value was 0.25. Species-specific PCR assays for $T$. brucei, T. congolense, and T. vivax was performed as previously reported $[15,16]$. For the purpose of subspecies identification of T. b. gambiense and T. b. rhodesiense, TgsGP (accession no. AM237444) specific (TgsGP-F: 5'-TCT TGA AGC CAA CAT GTC GG-3', and TgsGP-R: 5'-GAT CGC TCG TTT CCT GTC G-3') and SRA (accession no. Z37159) specific (SRA-F: 5'-CCG AAA GCG AAG CAC AGT T-3', and SRA-R: 5'-GGT TGC TGC CGA TAC TTG TT-3') primer pairs were designed in this study, respectively $[2,18]$. Since three subtypes of $T$. congolense, namely Savannah, Forest, and Kilifi, were reported, these subtypes specific PCR were also carried out according to the previous report $[13,16]$.

Mean hematocrit value of the sampled cattle was 26\% ranging from $11 \%$ to $45 \%$ (Fig. 2). This indicates that the nutritional state of each cattle appears to be poor during the early dry season, and herd mean hematocrit value was low in the sampled cattle. Nevertheless mean hematocrit value of MHC positive cattle (19.8\%) was significantly lower than that of MHC negative cattle (26.5\%) (Two-sample Student's $t$-test, $\mathrm{P}=0.0001)$. Thus the result proved that the trypanosome infection adversely impacted on cattle health condition, although no severe trypanosomosis was observed in the sampled cattle. In the MHC, 18 out of 203 cattle (8.9\%) showed parasitemia (Table 1) and another MHC positive case was identified as $T$. theileri infection. This result corresponds to the previous report whereby $7.1 \%$ of trypanosome prevalence was detected by microscopy in Tororo and Soroti districts, eastern Uganda [10]. Although MHC test is a simple and accurate diagnostic technique, it is not a suitable test for trypanosome species and subspecies identification. Therefore, total blood DNA of all cattle was subjected to PCR analysis. Among $18 \mathrm{MHC}$ positive samples, 5 were positive in $T$. brucei, 1 was positive in $T$. congolense Savanna subtype, and 1 was T. brucei/T. congolense Savanna double positive by PCR. Twelve MHC positive

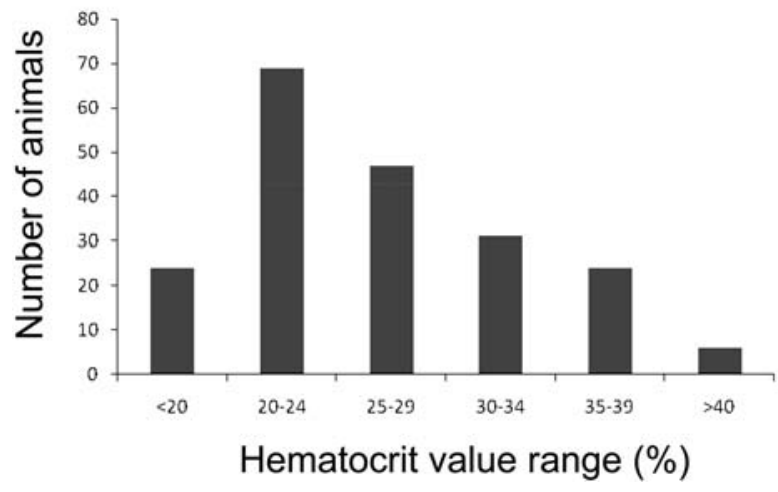

Fig. 2. Distribution of hematocrit values in the tested cattle population.

Table 1. Summary of the result (\%)

\begin{tabular}{ccccc}
\hline & & \multicolumn{3}{c}{ ELISA } \\
& & & + & - \\
& & 45.3 & 54.7 \\
\hline MHC & + & 8.9 & 5.9 & 3.0 \\
& - & 91.1 & 39.4 & 51.7 \\
\hline
\end{tabular}

The results were expressed as percentages of positive $(+)$ and negative ( - ) cattle in each method $(n=203)$.

samples were negative in PCR. This might indicate existence of different genotype(s) of trypanosome species which could not be detected by the PCR tests applied in this study. The discrepancy between MHC and PCR results also suggests either presence of inhibitory components for the PCR, existence of different genotypes of the parasite, or insufficient template DNA concentration [7]. Among 203 samples examine in this study, 34 samples were PCR positive (16.7\%) in which 3 were single positive for T. congolense, 28 were single positive for $T$. brucei, and 3 were double positive. All the PCR results were confirmed by the sequence analysis of the PCR products (data not shown). These PCR results are in accordance with previous reports whereby $T$. congolense Savana subtype is widely prevalent in the east Africa than the other two subtypes [1, 14]. Only 3 cattle samples were PCR positive for both $T$. congolense Savanna and T. b. brucei, whilst none of T. b. rhodesiense, T. b. gambiense, T. vivax, T. congolense Kilifi and Forest subtypes were detected by the specific PCR assays. Therefore, we concluded that all the T. brucei positive samples were actually $T$. $b$. brucei subspecies infections. Although cattle is a possible reservoir of $T$. $b$. rhodesiense, a causative agent of acute human African trypanosomosis (HAT), our result indicates that $T . b$. rhodesiense are not highly prevalent in cattle population in the studied area. Alternatively, this result may be due to sporadic epidemic of HAT [6]. It was surprising that none of the samples had T. vivax which is common in cattle in the eastern Uganda $[11,12]$. Since all the sampled cattle did not show obvious symptom of trypanosomosis, the authors concluded that cattle was infected with neither hemorrhagic $T$. vivax nor mild form $T$. vivax. 
Otherwise, a mild form $T$. vivax was under detectable level even in the PCR. Serological tests such as ELISA cannot differentiate between current and past infection [5], nevertheless they are useful diagnostic tools for determining prevalence of trypanosome infections. It has been previously reported that prevalence of trypanosomosis in cattle in Uganda is $11.9 \%$ under the intensive dairy system and $25 \%$ under the communal grazing systems [10]. With the need to keep up to date information, the current study has determined the prevalence of bovine trypanosomosis from a total of 203 samples collected from the cattle kept under the communal grazing systems in Butaleja district, Uganda. As a result, the seroprevalence of bovine trypanosomosis in the tested area was 45.3\% (92/203) (Table 1). In addition, 15 out of 34 PCR positive cattle samples were ELISA negative. This suggests that those 15 cattle were most recently infected with trypanosome parasites. While seropositive but PCR negative cattle were considered to be previously infected but already recovered. In conclusion, with a combination of MHC, ELISA and PCR, this study has clarified the prevalence and the major causative trypanosome species of bovine trypanosomosis in Butaleja district, eastern Uganda. Data obtained from this study will be useful for control of animal trypanosomosis in the studied area.

ACKNOWLEDGEMENTS. This study was supported by Grant-in-Aid for Scientific Research to SK, CS and NI, and by Research Grant Fellowship for Young Scientists to OT from the Japanese Society for the Promotion of Science (JSPS).

\section{REFERENCES}

1. Adams, E. R., Malele, I. I., Msangi, A. R. and Gibson, W. C. 2006. Trypanosome identification in wild tsetse populations in Tanzania using generic primers to amplify the ribosomal RNA ITS-1 region. Acta Trop. 100: 103-109.

2. Berberof, M., Perez-Morga, D. and Pays, E. 2001. A receptorlike flagellar pocket glycoprotein specific to Trypanosoma brucei gambiense. Mol. Biochem. Parasitol. 113: 127-138.

3. Courtin, D., Berthier, D., Thevenon, S., Dayo, G. K., Garcia, A. and Bucheton, B. 2008. Host genetics in African trypanosomiasis. Infect. Genet. Evol. 8: 229-238.

4. Davies, J. N. 1962. The cause of sleeping-sickness? Entebbe 1902-03. I. East Afr. Med. J. 39: 81-99.

5. de Almeida, P. P., Ndao, M., Van Meirvenne, N. and Geerts, S. 1998. Diagnostic evaluation of PCR on dried blood samples from goats experimentally infected with Trypanosoma brucei brucei. Acta Trop. 70: 269-276.

6. Enyaru, J. C., Matovu, E., Nerima, B., Akol, M. and Sebikali, C. 2006. Detection of T. b. rhodesiense trypanosomes in humans and domestic animals in south east Uganda by amplifi- cation of serum resistance-associated gene. Ann. New York Acad Sci. 1081: 311-319.

7. Gonzales, J. L., Jones, T. W., Picozzi, K. and Cuellar, H. R. 2003. Evaluation of a polymerase chain reaction assay for the diagnosis of bovine trypanosomiasis and epidemiological surveillance in Bolivia. Kinetoplastid Biol. Dis. 2: 8.

8. Leak, S. G. A. 1998. Tsetse biology and ecology: Their Role in the Epidemiology and control of Trypanosomosis, ed., CABI Publishing in association with the International Livestock Research Institute, Wallingford.

9. Magona, J. W., Greiner, M. and Mehlitz, D. 2000. Impact of tsetse control on the age-specific prevalence of trypanosomosis in village cattle in southeast Uganda. Trop. Anim. Health Prod. 32: 87-98.

10. Magona, J. W. and Mayende, J. S. 2002. Occurrence of concurrent trypanosomosis, theileriosis, anaplasmosis and helminthosis in Friesian, Zebu and Sahiwal cattle in Uganda. Onderstepoort J. Vet. Res. 69: 133-140.

11. Magona, J. W., Walubengo, J., Odiit, M., Okedi, L. A., Abila, P., Katabazi, B. K., Gidudu, A. M. and Olaho-Mukani, W. 2005. Implications of the re-invasion of Southeast Uganda by Glossina pallidipes on the epidemiology of bovine trypanosomosis. Vet. Parasitol. 128: 1-9.

12. Magona, J. W., Walubengo, J. and Odimin, J. T. 2008. Acute haemorrhagic syndrome of bovine trypanosomosis in Uganda. Acta Trop. 107: 186-191.

13. Majiwa, P. A., Maina, M., Waitumbi, J. N., Mihok, S. and Zweygarth, E. 1993. Trypanosoma (Nannomonas) congolense: molecular characterization of a new genotype from Tsavo, Kenya. Parasitology 106 ( Pt 2): 151-162.

14. Malele, I., Craske, L., Knight, C., Ferris, V., Njiru, Z., Hamilton, P., Lehane, S., Lehane, M. and Gibson, W. 2003. The use of specific and generic primers to identify trypanosome infections of wild tsetse flies in Tanzania by PCR. Infect. Genet. Evol. 3: 271-279.

15. Masake, R. A., Majiwa, P. A., Moloo, S. K., Makau, J. M., Njuguna, J. T., Maina, M., Kabata, J., ole-MoiYoi, O. K. and Nantulya, V. M. 1997. Sensitive and specific detection of Trypanosoma vivax using the polymerase chain reaction. Exp. Parasitol. 85: 193-205.

16. Masiga, D. K., Smyth, A. J., Hayes, P., Bromidge, T. J. and Gibson, W. C. 1992. Sensitive detection of trypanosomes in tsetse flies by DNA amplification. Int. J. Parasitol. 22: 909918.

17. OIE. 2001. Manual of Standards for Diagnostic Tests and Vaccines 2000, 4th ed., Office International des Epizooties, Paris.

18. Radwanska, M., Chamekh, M., Vanhamme, L., Claes, F., Magez, S., Magnus, E., de Baetselier, P., Büscher, P. and Pays, E. 2002. The serum resistance-associated gene as a diagnostic tool for the detection of Trypanosoma brucei rhodesiense. Am. J. Trop. Med. Hyg. 67: 684-690.

19. Van den Bossche, P. and Rowlands, G. J. 2001. The relationship between the parasitological prevalence of trypanosomal infections in cattle and herd average packed cell volume. Acta. Trop. 78: 163-170. 